\title{
EL FINANCIAMIENTO CLIMÁTICO INTERNACIONAL Y LOS DESAFÍOS DE SU CATALIZACIÓN. DEBATES SOBRE LA BRECHA ENTRE NECESIDADES CLIMÁTICAS Y FINANCIAMIENTO, DE BALI (2007) A PARÍS (2015)
}

María Elisa Brussa*

\section{Resumen}

El cambio climático es uno de los grandes desafíos de la agenda global. Para resolver estas cuestiones, desde el Plan de Acción de Bali en 2007 se ha consolidado a nivel global una arquitectura internacional de financiamiento climático altamente compleja, multi-institucional y multi-escalar.

Aunque el sistema funciona en muchos casos, varios aspectos obstaculizan la capacidad de catalizar el volumen completo de recursos, así como la implementación eficiente del financiamiento para lograr cambios transformacionales importantes, particularmente en aquellas "áreas difíciles de mitigar". Se sostiene así que el marco de un régimen policéntrico de la gobernanza financiera ambiental y de excesiva fragmentación organizacional e institucional del esquema de financiamiento de recursos climáticos, implica múltiples exigencias a nivel país que no necesariamente redundan en una asignación eficaz de las respuestas financieras al deterioro climático.

Para este trabajo se utilizó un método cualitativo. Para ello se recolectaron datos a través de la revisión bibliográfica de documentos y trabajos de investigación disponibles en organismos multilaterales de crédito y de organismos especializados de la cooperación ambiental internacional. Dichos datos fueron analizados mediante un análisis temático. Con este trabajo, se pretende contribuir a las discusiones sobre cooperación internacional climática.

Palabras claves: Finanzas climáticas - Cooperación internacional - Cambio climático - Gobernanza ambiental

\section{INTERNATIONAL CLIMATE FINANCE AND THE CHALLENGES OF CATALYZING IT. DEBATES ON THE GAP BETWEEN CLIMATE NEEDS AND FINANCING, FROM BALI (2007) TO PARIS (2015)}

\begin{abstract}
Climate change is one of the major challenges on the global agenda. To solve these issues, since the Bali Action Plan in 2007, a highly complex, multi-institutional and multi-scale international climate finance architecture has been consolidated at the global level.
\end{abstract}

\footnotetext{
* Licenciada en Relaciones Internacionales (Universidad Nacional de Rosario). Correo electrónico: ebrussa@gmail.com. https://orcid.org/0000-0002-8990-8677
} 
Although the system works in many cases, several aspects hinder the ability to catalyse the full volume of resources, as well as the efficient implementation of financing to achieve significant transformational change, particularly in those "hard-to-mitigate areas". It is argued that the framework of a polycentric environmental financial governance regime and the excessive organisational and institutional fragmentation of the climate finance scheme implies multiple demands at the country level that do not necessarily result in an effective allocation of financial responses to climate change.

A qualitative method was used for this work. Data was collected by reviewing the bibliography of documents and research papers available from multilateral credit organizations and specialized international environmental cooperation agencies. These data were analyzed through a thematic analysis. The aim of this work is to contribute to the discussions on international cooperation on climate.

Keywords: Climate finance - International cooperation - Climate change Environmental governance

TRABAJO RECIBIDO: 21/08/2020 - TRABAJO ACEPTADO: 15/11/2020 


\section{Introducción}

La Agenda 2030 para el Desarrollo Sostenible y el Acuerdo de París señalan el amplio compromiso internacional sobre la importancia de abordar los desafíos climáticos como parte de un enfoque holístico para el desarrollo sostenible y la reducción de la pobreza. El acceso al financiamiento climático es esencial para fomentar un desarrollo resiliente al clima y a una economía baja en carbono.

Este trabajo busca analizar las consecuencias del aumento creciente del financiamiento climático que emergen a partir del Plan de Acción de Bali (2007) hasta su impulso definitivo en el Acuerdo de París (2015). La proliferación de fondos climáticos visto como un "mosaico de fragmentación institucional" (Biermann, Pattberg, Van Asselt \& Zelli, 2009) y caracterizado por la duplicación, autoridad dispersa y regulaciones débiles (Kramarz \& Park, 2016) de las finanzas climáticas, sumado al "progresivo deterioro del ambiente y la incapacidad de mejorar la vulnerabilidad climática de los países menos desarrollados y en desarrollo, refleja en cierta forma la ineficacia en la reducción de la brecha entre las necesidades climáticas y el financiamiento verde" (Lohmann, 2009:529).

Es así que, en este trabajo, se busca responder las siguientes preguntas: ¿Cuál es la relación entre los actores principales de la gobernanza ambiental y los mecanismos financieros climáticos para cumplir el Acuerdo de París y las metas de los ODS? ¿Es suficiente la arquitectura actual de financiamiento para cumplir con el Acuerdo de París? ¿Cuáles son las opciones y los límites para el manejo de la fragmentación y la falta de coordinación institucional de los recursos financieros ambientales para una gestión eficaz de la brecha entre necesidades y finanzas climáticas?

Es decir, es la fragmentación (per se) a nivel mundial y nacional con su superposición de áreas e inconsistencias en los procedimientos de acceso y gestión de la financiación (Amerasinghe, Thwaites, Larsen, \& Ballesteros, 2017) o la falta de coordinación entre organizaciones ambientales y mecanismos financieros, regulación en la contabilidad y monitoreo de la efectividad global de las respuestas, las que obstruyen la posibilidad de pensar nuevos enfoques. Lohmnan (2009:530) lo ejemplifica con

la indiferencia en la contabilidad del carbono sobre dónde o cómo se realizan los recortes de emisiones (...); su combinación de reducciones y compensaciones conduce a un conjunto de probabilidades de incertidumbre, ignorancia e indeterminación; y su enfoque para lograr la eficiencia a corto plazo obstruye el pensamiento social sobre las direcciones a largo plazo y los inconvenientes de tener que controlar los efectos geográficamente distantes.

Este trabajo está organizado de la siguiente manera. En primer lugar, se llevará a cabo una descripción del despliegue del financiamiento climático y de los mecanismos para su movilización e implementación, para luego abordar algunos aspectos que actúan como barreras a iniciativas eficientes y alineadas con los objetivos establecidos en el Acuerdo de París. El financiamiento climático internacional es un subconjunto de financiamiento verde que se refiere a inversiones específicamente en actividades de mitigación y adaptación al cambio climático que involucran principalmente financiamiento público y el apalancamiento de la financiación privada en los países en 
desarrollo (Warren, 2019).

Es así que este artículo se introduce en el debate sobre la arquitectura de la gobernanza ambiental, que incluye el nuevo institucionalismo (Ostrom, 1990; Biermann \& Pattberg, 2008; Biermann et al. 2009; Najam, Adil y Halle, 2010) y se vincula con debates actuales sobre la cuestión de la efectividad, legitimidad y eficiencia de los nuevos instrumentos y mecanismos de la gobernanza financiera ambiental global.

En segundo lugar, como forma de "enmarcar" (Callon, 1999) posibles modalidades de gestión de la fragmentación y de sistematizar la densidad (Barnes, 1954) y conexidad de las barreras, se exploran opciones para la gestión organizacional e institucional de la fragmentación, proponiendo algunos ejes de análisis, las Políticas y los Proyectos financieros, el Comercio Internacional climático y los Mercados Financieros Verdes, ámbitos sinérgicos y transversales a todas las etapas de implementación de los fondos climáticos.

Asimismo, los datos surgen de la revisión bibliográfica disponible de grupos económicos multilaterales como el Comité de Ayuda al Desarrollo de la Organización para la Cooperación y el Desarrollo Económicos (OCDE), el Reporte conjunto de los Bancos multilaterales de Desarrollo y de organizaciones encargadas de monitorear los flujos de finanzas climáticas como el Climate Policy Initiative, así como los documentos y trabajos surgidos principalmente de las Convención Marco de Naciones Unidas sobre el Cambio Climático.

\section{Configuraciones del financiamiento climático}

El presente apartado está dedicado a examinar las condiciones de emergencia del financiamiento climático mundial, así como los principales dispositivos financieros que se fueron constituyendo dando lugar a la actual fragmentación de la gobernanza ambiental $^{1}$ internacional. Proponemos aquí, un uso más restrictivo del término de gobernanza ambiental asociada a la definición de Young (1999:11) como "el esfuerzo combinado de regímenes internacionales y transnacionales", que gobiernan el uso humano de los recursos naturales y los servicios ambientales y que alude a la conformación de una nueva arquitectura de gobernanza ambiental a causa de la aparición de nuevos actores, mecanismos e instrumentos con el objetivo de resolver o dar respuestas más eficaces a los problemas surgidos de las llamadas sociedades de riesgo o posmodernas (Beck, 2000).

Esta emergente gobernanza financiera ambiental se caracteriza por una creciente segmentación de diferentes capas de actores que implementan clusters de reglas "entre capas de autoridades supranacionales, internacionales, nacionales y subnacionales (gobernanza multinivel) y horizontalmente entre diferentes sistemas paralelos de regulación ejecutados por diferentes grupos de actores (gobernanza multipolar)" (Biermann \& Pattberg, 2008:284). Es decir, un "mosaico internacional de instituciones

\footnotetext{
${ }^{1}$ A pesar del origen bastante reciente del término de gobernanza ambiental este concepto tiene como antecedentes los estudios de cooperación ambiental internacional de décadas anteriores, sobre la investigación de regímenes ambientales (Krasner, 1983, Mitchell, 2003). Una de las definiciones de régimen internacional es la de Krasner (1983: 2) quien propone que un "régimen internacional es un conjunto implícito o explícito de principios, normas, reglas y procedimientos de toma de decisiones, alrededor de los cuales las expectativas de los actores convergen en un área de las relaciones internacionales".
} 
que son diferentes en su carácter (organizaciones, regímenes y normas implícitas), sus circunscripciones (públicas y privadas), y su alcance espacial (de bilateral a global), con una temática predeterminada". (Biermann et al., 2009:16).

Como motor de la configuración de este escenario, fue esencial la creciente preocupación por las emisiones antropogénicas de gases de efecto invernadero y los impactos de estas, que culminó con el establecimiento del Panel Intergubernamental para el Cambio Climático (IPCC) conjuntamente por el Programa de las Naciones Unidas para el Medio Ambiente (PNUMA) y la Organización Meteorológica Mundial (OMM) y en paralelo a la conformación de una nueva racionalidad del tratamiento de la cuestión ambiental basada en la mercantilización de la atmósfera y de la naturaleza y de la concepción de "capital natural" "vinculada a la noción más conocida de Desarrollo Sostenible (Seoane, 2017).

Si nos focalizamos en las nuevas instituciones de la gobernanza ambiental, la Convención Marco de Naciones Unidas sobre el Cambio climático (1992) constituyó el punto de consenso de evaluaciones sobre el cambio del clima y sus fuentes antropógenas, así como el inicio de una red global de políticas ambientales ligadas a la medición de los límites de contaminación y de la obligación de financiar acciones de "adaptación"3 y "mitigación"4 para cumplir con los objetivos propuestos en el Acuerdo de París. Así es que "el análisis del financiamiento para enfrentar el cambio climático debe enmarcarse en los acuerdos y negociaciones internacionales para estabilizar las emisiones de gases de efecto invernadero en la atmósfera y reducir la vulnerabilidad y los riesgos frente a la variabilidad climática" (Cabral y Bowling, 2014:15) así como en los nuevos actores y mecanismos que surgieron de estos.

Fue entonces bajo el paraguas de la CMNUCC, que los países del Anexo $\mathrm{II}^{5}$, debieron proporcionar financiamiento relacionado con el clima a los países en desarrollo. (CMNUCC, 1992:18). La CMNUCC estableció la responsabilidad financiera clara de los países ricos contaminantes, al incluirlos en dicho Anexo como responsables de financiar la descarbonización de la sociedad y la adaptación al cambio climático en los países empobrecidos.

Es a partir del Acuerdo de Kioto que el proceso global de financiarización de la crisis climática toma forma como soluciones de mercado del carbono, evidenciándose una simultaneidad en la formación de mercados de servicios ambientales y en la expansión del proceso de financiarización de las últimas décadas (Harvey, 2005). Pero es

\footnotetext{
${ }^{2}$ En este sentido, para este concepto se señalan los trabajos, particularmente de Daly (1989) que desarrolla el documento Nuestro Futuro Común (Our Common Future, en inglés) de 1987 de la ONU y el de Nicholas Georgescu-Roegen de 1971 que postula la perspectiva de la "bioeconomía". (Constanza\& Daly, 1992).

${ }^{3}$ La adaptación se relaciona con iniciativas que buscan realizar ajustes en los sistemas naturales o humanos en respuesta a estímulos climáticos reales o esperados, disminuyendo así el daño que ocasionan o aprovechando sus beneficios.

${ }^{4}$ La mitigación busca desarrollar medidas para reducir directamente el nivel de emisiones, además de preservar y mejorar los sumideros y reservorios de GEI.

${ }^{5}$ Partes Anexo I: incluye a los países industrializados que eran miembros de la OCDE en 1992, más países con economías en transición (las Partes de EIT), incluyendo la Federación Rusa, los Estados Bálticos, y varios Estados Europeos Centrales y Occidentales. Partes Anexo II: Consiste en países del Anexo I miembros de la OCDE, sin las Partes EIT. Están obligados a brindar recursos financieros para permitir a los países en desarrollo llevar a cabo actividades para la reducción de emisiones bajo la Convención y a ayudarles a adaptarse a los efectos adversos del cambio climático. Adicionalmente, deben "tomar todos los pasos practicables" para promover el desarrollo y transferencia de tecnologías ambientalmente amigables a las Partes EIT y países en desarrollo.
} 
con la gobernanza ambiental nacida del Acuerdo de París ${ }^{6}$ que se impulsan las más conocidas iniciativas multilaterales $\mathrm{y}$ bilaterales sobre el cambio climático, paradójicamente en sintonía con una fase de compromisos de reducciones más flexibles y voluntarias. El Acuerdo de París a través de la decisión 1/CP.21 consensuó el objetivo básico global de mantener el aumento de la temperatura media mundial debajo de $2^{\circ} \mathrm{C}$ con respecto a los niveles preindustriales ${ }^{7}$. En la práctica significó un afianzamiento de la operacionalización del desarrollo sostenible (Agenda 2030 de ODS) y de la cuantificación de los riesgos ambientales y de la reducción de emisiones de GEI como exigencia para la asignación de flujos financieros ambientales.

De la misma forma se fueron consensuando definiciones de las finanzas verdes como aquellas referidas al "financiamiento local, nacional o transnacional, proveniente de fuentes de financiamiento públicas, privadas y alternativas, que busca respaldar las acciones de mitigación y adaptación que abordarán el cambio climático" (CMNUCC, 2016). Por su parte, OCDE (2015) define las finanzas climáticas como "los flujos de capital que buscan el desarrollo bajo en carbono y resilientes al clima, los cuales pueden ser públicos o privados, concesionados y no concesionados".

Como forma de sintetizar el entramado de la estructura de financiamiento climático y de la multiplicidad de organismos para su implementación, desarrollaremos una descripción del tratamiento de los diferentes dominios de la gobernanza ambiental global - el cambio climático, la diversidad biológica, las energías renovables - como demostración de la fragmentación institucional de los fondos ambientales o la existencia de regímenes específicos como "redes parciales" (Barnes, 1954) de dominios particulares.

Es así como en el marco de la Cumbre de la Tierra (1992), se crea la Iniciativa de las Instituciones Financieras del PNUMA, o (PNUMA-IF, o en inglés UNEP-FI) como una "plataforma de asociación entre las Naciones Unidas y el sector financiero global" (UNEP-FI, s/f; 2020). También en el marco de dicha cumbre, el Convenio sobre la Diversidad Biológica (CDB), que focaliza en la biodiversidad y en el enfoque por ecosistemas $^{8}$ implementa políticas concretas de financiamiento de servicios y bienes ambientales. Enfoque que se enriquece a partir de la COP 11 (Montreal) y de la COP 13 (Bali) con la "reducción de emisiones por deforestación en países en desarrollo y acercamientos para estimular la acción sobre el tema" en el marco de la mitigación del cambio climático. En ellas nacen las iniciativas multilaterales y bilaterales de proyectos o mecanismos para la Reducción de Emisiones causadas por la Deforestación y la Degradación de los Bosques (REDD). Para que los países se preparen en el desarrollo de mecanismos REDD, se propuso un enfoque de implementación por fases de acuerdo con

\footnotetext{
${ }^{6}$ En realidad se operacionaliza en la Agenda de Acción de Addis Abeba (2015) que establece el marco mundial para financiar el desarrollo después de 2015 para avanzar en la movilización de recursos financieros y técnicos para el desarrollo procedentes de un mayor número de agentes

7 Según lo planteado por el Panel Intergubernamental que se traslada al Acuerdo de París, para el año 2050 la temperatura media del planeta no puede aumentar más de $2{ }^{\circ} \mathrm{C}$ respecto a la temperatura de antes de la Revolución Industrial. Pero además de no superar los $2{ }^{\circ} \mathrm{C}$, el Acuerdo fija que el aumento de temperatura debe mantenerse lo más cerca posible a $1,5{ }^{\circ} \mathrm{C}$, para evitar los peores impactos del cambio climático. Las predicciones de los científicos del Grupo Intergubernamental sobre el Cambio Climático (IPCC por sus siglas en inglés) apuntan a que alcanzaremos y superaremos esta temperatura entre 2030 y 2050. De ahí surge que la agenda de la Agenda Sostenible sea a 2030.

${ }^{8}$ Este tópico también da lugar a otras convenciones como: la Convención sobre la conservación de las especies migratorias de animales silvestres; la Convención de Ramsar; la Convención de Naciones Unidas de Lucha contra la Desertificación.
} 
las condiciones específicas de cada país. En la fase inicial, conocida como REDD readiness, se desarrollarán las estrategias a nivel nacional. En la fase dos, se implementan las políticas y medidas que posibilitan REDD; además de que se promueve la ampliación de las inversiones. En la fase tres, los fondos y mecanismos de mercado realizarán pagos por resultados vinculados con controlar emisiones, medir, elaborar informes y verificar.

Sin embargo, es a partir de la Conferencia de las Partes (COP) de la CMNUCC que aparecen los fondos climáticos específicos dando lugar fundamentalmente a dos tipos de financiamiento climático internacional que determinan una especificidad de actores y mecanismos.

1) Financiación climática dedicada o específica: se refiere a la proporcionada por gobiernos (en su mayoría de la OCDE) en condiciones favorables y con el objetivo explícito de lograr un desarrollo resiliente bajo en carbono. Son ejemplos de los mismos los Fondos Especializados del Clima, que aparecen como vehículos de movilización de recursos de sus donantes, pero que dependen muchas veces, de otras entidades ejecutoras para desembolsar dichos fondos. Como señalamos anteriormente, estos nacen en el marco de la CMNUCC. El primero en crearse fue el Fondo para el Medio Ambiente Mundial (FMAM en inglés GEF) que ejerce también como mecanismo financiero del Convenio sobre la Diversidad Biológica (CDB) y de la Convención Marco de las Naciones Unidas sobre el Cambio Climático (CMNUCC). A su vez, actualmente, administra el Fondo para Países menos Desarrollados (LDCF en inglés), el Fondo Especial para el Cambio Climático (SCCF en inglés) y el Fondo para la Aplicación del Protocolo de Nagoya (NPIF en inglés). En la COP16 de Cancún (2010) se estableció el Fondo Verde para el Clima. Los fondos climáticos multilaterales participaron en el financiamiento global, en 2017, con 435 millones de dólares, (88 millones menos que en 2016) siendo desplazados por la participación cada vez mayor de los Bancos Multilaterales de Crédito (CPI, 2018).

Tabla 1: Panorama de fondos multilaterales del clima

\begin{tabular}{|l|l|}
\hline FONDOS climáticos de & Fondo de Adaptación (AF) \\
la CMNUCC & Fondo para los Países menos Desarrollados (LDCF) \\
& Fondo Especial para el Cambio Climático (SCCF) ${ }^{11}$ \\
& Fondo Verde del Clima (GCF) \\
& Fondo para el Medio Ambiente Mundial \\
& $(\mathrm{GEF} / 6)$ \\
\hline
\end{tabular}

\footnotetext{
${ }^{9} \mathrm{https}$ ///www.adaptation-fund.org/apply-funding/policies-guidelines/.

${ }^{10}$ El Fondo para los Países menos Desarrollados (LDCF) se estableció en la (COP 7) de Marrakech (2001) El Fondo Mundial para el Medio Ambiente (GEF) administra el LDCF. El Banco Mundial actúa como el fiduciario financiero del GEF. Este fondo implementa proyectos urgentes de adaptación.

${ }^{11} \mathrm{Al}$ igual que el LDCF, no proporciona acceso directo. Por lo tanto, las solicitudes tienen que presentarse en oficinas de gobierno en cooperación con organismos implementadores como: FAO, UNDP, bancos multilaterales de desarrollo [BMD]) que están acreditadas con el GEF.

${ }^{12} \mathrm{Su}$ enfoque sectorial se basa en proyectos de acuerdo con las estrategias y planes climáticos (tales como las estrategias de desarrollo bajo en emisiones, las Acciones Nacionales de Mitigación Apropiadas [NAMA], los NAPA, los Planes Nacionales de Adaptación. Disponible en: https://www.greenclimate.fund/about.
} 


\begin{tabular}{|c|c|}
\hline $\begin{array}{l}\text { Instituciones } \\
\text { financieras que no son } \\
\text { de la CMNUCC: }\end{array}$ & 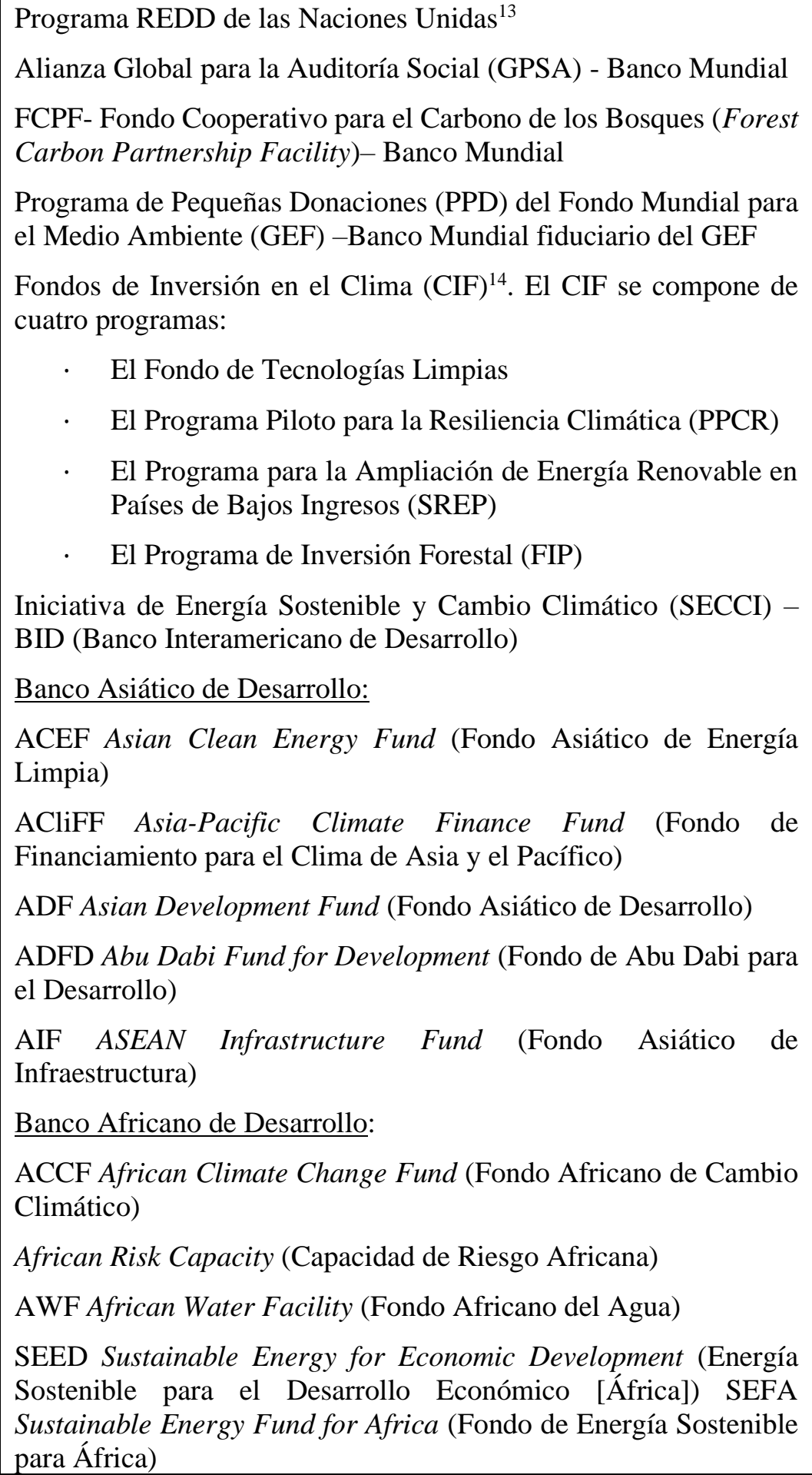 \\
\hline
\end{tabular}

Fuente: Creación propia

2) Financiación del desarrollo relacionada con el clima: se refiere a la proporcionada por fuentes multilaterales y bilaterales. Estas instituciones actúan como agencias

\footnotetext{
${ }^{13}$ Las organizaciones participantes de las UN actúan como agencias ejecutoras. Usualmente se solicita en una oficina del país del PNUD.

${ }^{14}$ El Banco Internacional de Reconstrucción y Fomento (BIRF) del Grupo del Banco Mundial se desempeña como el fiduciario del Fondos de Inversión en el Clima (CIF).
} 
implementadoras que a su vez pueden involucrar a otra capa de entidades receptoras. Estos diferentes niveles de capas múltiples y superpuestas resaltan la fragmentación del sector y el aumento de barreras para el acceso e implementación de los recursos financieros. Entonces cuanto más amplio sea el dominio de acción del Fondo, es más probable que implique más capas de agencias y esferas temáticas ambientales y no ambientales e instituciones asociadas así al modelo de la cebolla, dando lugar a una idea de clustering en base a dominios específicos según los tipos de medidas de financiamiento y de conexidad o de densidad de vínculos en base a los distintos órdenes o niveles de capas. Es así que, por ejemplo, la financiación de la adaptación utiliza para su administración las redes de organizaciones específicas de Naciones Unidas como el Programa de las Naciones Unidas para el Desarrollo (PNUD), el PNUMA, el Programa Mundial de Alimentos (PMA). De la misma forma, los bancos multilaterales de crédito como el Banco Mundial (BM), en su rol de agencia implementadora, tienen un papel importante en la inversión de fondos administrados como el Fondo para una Tecnología Limpia (CTF), el Fondo de Inversión Forestal (FIP), el Programa Piloto sobre capacidad de adaptación del Cambio Climático (PPCR) y el Programa destinado al fomento de las energías renovables (SREP). Se suman a la lista de agencias administradoras de fondos, la Corporación Andina de Fomento (CAF) como mayor financiador multilateral en infraestructura "verde" y el BID, entre otras.

En cuanto a los actores bilaterales más importantes en el marco del financiamiento climático se destaca la Agencia Francesa de Desarrollo (AFD), el Banco de Desarrollo de Alemania (KFw/GIZ), la agencia noruega (NORAD) y la Agencia de Cooperación Internacional de Japón (JICA). Estos últimos ejemplos indican que la financiación climática y la cooperación para el desarrollo (AOD) se desembolsan a través de canales similares y que muchas veces pueden ser indicativos de un redireccionamiento de fondos a otras prioridades de desarrollo relevantes. De hecho, existe un debate de larga data sobre si la adaptación y mitigación climáticas deben tratarse por separado a la cooperación al desarrollo o través de conceptos unificadores como "desarrollo bajo en carbono" o "desarrollo resiliente al clima" para cerrar esa brecha entre ambos tipos de financiamiento.

\section{Financiamiento climático versus necesidades climáticas}

El alcance y la escala de los cambios necesarios para que las economías y las sociedades logren una resiliencia baja en carbono son inmensos y requieren una transformación sustancial de las economías involucradas. Ejemplo de esto último es el compromiso de movilizar conjuntamente USD 100 mil millones por año en flujos internacionales de financiamiento climático a países en desarrollo (OCDE, 2015). Si bien la financiación climática alcanzó niveles récord, la acción aún está muy por debajo de lo que se necesita en un escenario de $1.5^{\circ} \mathrm{C}$. Dicha constatación hizo que se desarrollara el concepto de "presupuesto de carbono", que no es más que la cantidad de emisiones de dióxido de carbono que podemos emitir mientras todavía tenemos una posibilidad de limitar el aumento de la temperatura global a 2 grados por encima de los niveles preindustriales.

En la relación entre los componentes de ambiente y finanzas, el elemento que adquiere mayor importancia es el cambio climático, es decir, el calentamiento global asociado a costos impuestos a las futuras generaciones. Para resolver esta paradoja y, 
especialmente, para establecer los incentivos apropiados, los mercados financieros aparecieron como instrumentos para valorar (en el sentido de estimar el valor económico y financiero) los objetivos y compromisos acordados en la Cumbre de París. Es así como la relación entre medio ambiente y finanzas se ve reflejada en la utilización de diferentes metodologías para la tipificación de riesgos climáticos y la cuantificación de las necesidades climáticas, es decir, los recursos necesarios para abordar la mitigación y adaptación al cambio climático como las que recomienda el grupo de trabajo (TCFD, o Task Force on Climate-related Financial Disclosure) del mencionado FSB. Sin tomar en cuenta los riesgos físicos y fiduciarios, se puede medir el riesgo en transición y estimarlo en un aproximado de inversión requerida para lograr el rango de transición bajo en carbono en USD 1.6 billones a USD 3.8 billones anuales entre 2016 y 2050, solo para inversiones en el sistema de energía del lado del suministro (IPCC, 2018). En esta misma dirección de identificar activos e identificar sus riesgos de transición es que se llevan a cabo mediciones del grado de exposición de mercados que basan su modelo de negocio en recursos energéticos fósiles. A nivel mundial, estos cambios de transición prevén una reducción de las inversiones en combustibles fósiles en aproximadamente un 30\% y una ampliación de las inversiones en eficiencia energética y generación de energía baja en carbono, incluidas las energías renovables, en aproximadamente el mismo porcentaje. Actualmente las iniciativas de mitigación ${ }^{15}$ se llevan el $94 \%$ del financiamiento y el resto va a la adaptación ${ }^{16}$. El $65 \%$ de estos proyectos están destinados al sector energético, en especial los de ahorro y eficiencia de energía y energías renovables (CPI, 2018).

Se estima que el financiamiento de países pertenecientes a la OCDE hacia los países en desarrollo es de aproximadamente USD 356 mil millones (promedio anual 2017-2018). Estos números incluyen el financiamiento climático privado, la financiación climática pública nacional y la financiación climática pública internacional y representa menos del $10 \%$ de las necesidades totales de inversión. Esto significa que alrededor del 90\% de las inversiones habituales, aún se desarrollan, sin contemplar el desarrollo relacionado con el clima.

El eje central de la gestión del financiamiento climático es el vínculo entre el nivel de emisiones y las diferentes estrategias de contabilidad verde, es decir, en relación con los riesgos del cambio climático y la huella de carbono y los compromisos con objetivos cuantificables de reducción de la exposición a activos causantes de emisiones de gases de efecto invernadero (GEI).

A la vez, este vínculo entre el grado de responsabilidad de emisiones y su correlato en el financiamiento adquiere otra perspectiva a partir del binomio ambiente/geopolítica. Es así que el monitoreo de los flujos financieros según el nivel de emisiones por regiones presenta una gran asimetría entre emisiones y la vulnerabilidad de los impactos climáticos. En el caso de América Latina y el Caribe, las emisiones totales representan solo el 8,3\% de las emisiones mundiales, pero, al mismo tiempo, la región es particularmente vulnerable al impacto del cambio climático ${ }^{17}$ debido a sus características

\footnotetext{
${ }^{15}$ El financiamiento orientado a la mitigación se orienta a promover la eficiencia energética, el desarrollo de energías renovables, la preservación de los bosques o infraestructura urbana sostenible.

${ }^{16}$ La financiación orientada a la adaptación puede incluir inversiones en infraestructura para reducir riesgos de desastres o apoyo financiero para desarrollo agrícola o actividades de gestión del agua.

${ }^{17} \mathrm{Al}$ mismo tiempo que se siguen sosteniendo los mismos patrones de inserción asimétrica internacional como países productores y exportadores de productos primarios, a la vez la agricultura es una de las actividades que podría sufrir impactos directos a causa del cambio climático, por el aumento de la
} 
geográficas, climáticas, socioeconómicas y demográficas (CEPAL, 2015). El tema de las responsabilidades del consumo y de las emisiones sumado a la escasez o disponibilidad de recursos naturales termina conformando lo que se llama una "ambientalización de la geopolítica" (Nogué Font y Vicente Rufí, 2001: 200), o "geopolítica del ambiente" (Estenssoro Saavedra, 2013) que determina de cierta forma la orientación de las políticas de mitigación, adaptación o conservación por parte de los países receptores de fondos y condiciona el destino y en el volumen de los flujos de financiamiento de políticas.

En el espacio regional latinoamericano los flujos financieros climáticos alcanzaron un promedio anual de USD 20.500 millones entre 2013 y 2017 (CEPAL, 2019). Contrario a la tendencia mundial, el flujo de estos recursos ha sido creciente en la región, concentrando el 9\% del financiamiento total. El financiamiento para el clima en América Latina está muy concentrado, y Brasil y México reciben casi la mitad de los fondos para la región. La ausencia de criterios de asignación a nivel global y la multiplicidad de factores para su acceso contribuye a una sobrerrepresentación de actores financieros en determinados países y sectores y subrepresentación en otros. Por ejemplo, las actividades de mitigación, como la protección de los bosques y la reforestación, reciben seis veces más fondos que las actividades de adaptación de los fondos multilaterales para el clima, con USD 3200 millones y USD 500 millones respectivamente. En cuanto a tipo de políticas financiadas en la región, la adaptación ha recibido en: política ambiental y gestión administrativa (25\%), prevención de desastres $(24 \%)$, agua y saneamiento (22\%) y agricultura (8\%). En mitigación (general) los sectores más relevantes han sido generación de energía y fuentes renovables (40\%), eficiencia energética (11\%) y transporte y almacenamiento (25\%).

\section{Gestionar la coordinación de las finanzas climáticas}

La coordinación del diseño y planificación del financiamiento internacional es necesario para impulsar y lograr catalizar tanto en volumen como en eficacia el financiamiento climático internacional y generar una verdadera transformación de las economías y de las políticas climáticas nacionales. Pero también se requiere una mayor comprensión de las políticas de transición y sus dimensiones políticas, sociales y económicas y cuáles son los impactos en cada una de ellas.

Hay un incipiente número de estudios que se están dedicando a analizar las características emergentes de la estructura de gestión del financiamiento climático para maximizar el impacto transformador de las finanzas sostenibles. Es así que a través de la descripción de la emergencia de la gobernanza financiera climática podemos visibilizar sus principales características como los diversos enfoques epistemológicos producto del grado de riqueza y diversidad conceptual, el problema de la estructura de las instituciones, los marcos estáticos y normalizadores del acceso metodológico.

Como ya hemos señalado, la falta de la coordinación institucional del financiamiento climático puede producirse tanto horizontalmente (entre unidades

temperatura y la variabilidad de la precipitación que afectan el rendimiento de los cultivos, el ingreso agrícola y la producción de autoconsumo, lo que generaría fuertes presiones sobre la disponibilidad y los precios de los productos agrícolas (Mendelsohn, Dinar \& Sanghi, 2001). Irónicamente, este sector también es uno de los mayores contribuyentes al cambio climático, con una participación del $11 \%$ de las emisiones mundiales de gases de efecto invernadero. 
subnacionales) como verticalmente (entre entidades internacionales, nacionales y subnacionales) (Nakhooda y Jha, 2014) y en el transcurso de las diferentes etapas: movilización y contabilidad, la arquitectura de fondos, asignación y canales de entrega.

En primer lugar, se hace evidente el desacuerdo sobre quién debería controlar estos fondos en torno a la discusión sobre las teorías de las relaciones internacionales acerca de estructuras policéntricas (Ostrom 2010) o centralizadas, ejemplo que se refleja en la cantidad de fondos por fuera de la estructura de la CMNUCC, que a la vez no son fondos dedicados exclusivamente al clima ${ }^{18}$, lo que puede definirse "como conjunto de regímenes específicos" (Keohane \& Victor, 2011:7) o regímenes específicos según las categorías principales de financiación climática: mitigación o adaptación. Ambas categorías no solo difieren en sus objetivos sino también en el tipo de características y el énfasis diferenciado de la financiación que promueven. La financiación de la mitigación busca contribuir al objetivo de reducir las emisiones globales, mientras que la financiación de la adaptación se centra en la gestión localizada de los efectos del cambio climático. El financiamiento de la mitigación comúnmente involucra el uso de préstamos y presenta un mayor potencial para la participación del sector privado, mientras que la adaptación se desembolsa generalmente en forma de subvenciones $\mathrm{y}$ ha tenido dificultades para atraer la participación del sector privado (Locatelli, Fedele, Fayolle \& Baglee, 2016). Como resultado, los países receptores condicionados por la especificidad de las categorías de movilización de recursos financieros fueron diagramando planificaciones del financiamiento recurrentes y homogéneas, por sectores como las NDS (en inglés, National Development Strategy) o Estrategias Nacionales de Desarrollo o las Políticas Nacionales de Cambio Climático (PNCC).

$\mathrm{Si}$ bien dichas estrategias buscan producir un mayor ordenamiento de metodologías para la movilización de fondos específicos, vinculadas primordialmente al ciclo actual de NDC, no se evidencian prácticas existentes de vinculación estratégica de problemas a través de diferentes tipos de actores para maximizar sus intereses en un entorno fragmentado de mecanismos institucionales que permitan operativizar los objetivos propuestos.

En cuanto a la contabilidad, parecen necesarias definiciones más estandarizadas del financiamiento climático como una forma de monitorear la movilización de recursos (Roberts \& Weikmans, 2017). Una modalidad utilizada es contar con un Sistema de Financiamiento Climático Nacional como alternativa viable para facilitar el seguimiento y la contabilidad, es decir, conocer en qué actividades están siendo utilizados los recursos existentes sobre cambio climático.

En este sentido, la CEPAL (Cabral y Bowling, 2014), advierte la dificultad de obtener información pública con respecto a los flujos climáticos que sean relevados específicamente por los países, y que no se cuente solo con aquella otorgada por los bancos de desarrollo. Muchos presupuestos nacionales no contemplan ni siquiera un apartado para procesarla. Es notable como el seguimiento del financiamiento climático de un país determinado muchas veces aparece condicionado a los datos de los bancos multilaterales de crédito (principales agencias implementadoras de la región), los cuales

\footnotetext{
${ }^{18}$ El campo de la financiación climática, como campo de poder estratégico se ha desarrollado a partir de regímenes ambientales internacionales específicos separados de la cooperación al desarrollo de los que provienen los fondos de los bancos multilaterales de crédito por ejemplo. Por lo tanto, son campos que no han estado integrados, lo que agrega más confusión a un panorama ya de por sí fragmentado de las finanzas sostenibles.
} 
a su vez cuentan con criterios diferenciados de contabilidad de las líneas temáticas que cada uno desarrolla. En algunos casos, el criterio de la financiación de proyectos es el cambio climático como objetivo principal y en otros el criterio es el proyecto donde la finalidad principal es otra, pero tienen componentes ambientales o son diseñados como proyectos multifocales, sinérgicos tanto con las medidas de mitigación como de adaptación, tal es el caso de los proyectos de infraestructura y saneamiento o en transporte público.

En esta misma etapa de contabilidad verde, muchos países han introducido en su sistema de planificación indicadores y metas para la evaluación de los objetivos específicos que considera el país. Pero es un esquema preliminar a un sistema de monitoreo, reporte y verificación (MRV) de las finanzas climáticas para entender hacia dónde están fluyendo los recursos financieros, para qué propósito y qué tan efectivamente mitigan las emisiones de GEI y/o ayudan a contribuir a cerrar la brecha financiera de adaptación. Ampliar las redes de monitoreo significa poder cuantificar económicamente los impactos del cambio climático y la implementación de medidas de adaptación necesarias. Recientes estudios (Glemarec, 2011; OCDE, 2018; Daly, 1989) vienen promoviendo la implementación de Metodologías de Seguimiento de finanzas climáticas (MDB).

En lo que respecta a la asignación, no existe una coordinación general sino un sistema de autocontrol donde los fondos van analizando donde se están realizando las asignaciones de otros fondos para así ir revisando sus propias asignaciones. La creación del Comité Permanente de Finanzas CMNUCC (COP16) entre sus objetivos, buscó abordar el problema de la ausencia de reglas contables acordadas internacionalmente que permitan evaluaciones generales del progreso (hacia el objetivo de movilizar conjuntamente USD 100 mil millones para 2020) y cualquier comparación significativa de desempeño entre países. Este Comité produce evaluaciones bienales de los flujos financieros basando sus datos en informes nacionales. Sin embargo, estos informes no logran superar el desnivel de datos de los informes nacionales, que ponen entredicho también los sistemas de seguimiento de finanzas climáticas, por ejemplo, el sistema de marcadores de Río ${ }^{19}$ y la de componentes climáticos ${ }^{20}$.

Es por eso que varios países con el objetivo de gestionar y a la vez coordinar los criterios de asignación de la financiación climática están desarrollando mecanismos de

\footnotetext{
${ }^{19}$ Los marcadores de Río son mecanismos de cuantificación aproximada de la financiación climática y proporcionan una indicación del grado de integración de las temáticas y objetivos climáticos en las carteras de cooperación. Tiene un sistema de puntuación de tres niveles: Principal (2) cuando el objetivo (mitigación del cambio climático o adaptación) se declara explícitamente como fundamental en el diseño de, o la motivación para, la actividad; Significativo (1) cuando el objetivo (cambio climático mitigación o adaptación) se establece explícitamente pero no es el impulsor fundamental o motivación para emprenderlo; No dirigido (0), lo que significa que la actividad fue examinada y de la cual se refleja que no apunta al objetivo (mitigación del cambio climático o adaptación) de cualquier manera significativa. (OCDE, 2018) ${ }^{20}$ La metodología de marcadores climáticos OCDE-CAD hace hincapié en los componentes climáticos y es implementado principalmente por los bancos multilaterales de desarrollo. La metodología identifica los componentes de un proyecto que contribuye directamente o promueven la adaptación y / o mitigación. Es una medida esencialmente cuantitativa. Los componentes se calculan de acuerdo con la articulación de la Metodología para el seguimiento de las finanzas (MDB) de mitigación climática y de la Metodología para el seguimiento de Finanzas (MDB) de Adaptación del clima. Las definiciones MDB y los marcadores de Río de mitigación y adaptación al cambio climático pueden usarse conjuntamente (OCDE, 2018).
} 
coordinación institucionalizados. El enfoque de agrupamiento ${ }^{21}$ se basa en el supuesto de que el número actual de instituciones ambientales internacionales es demasiado grande para ser posiblemente eficiente a nivel de la gestión nacional del financiamiento. Tales medidas podrían incluir agrupar operaciones y presupuestos; para coordinar o mejorar la transparencia y la participación para minimizar la superposición y la fragmentación institucional (Ivanova, 2007).

La necesidad de coordinación a nivel nacional también viene impulsada desde el ámbito internacional: en primer lugar, las Medidas de Mitigación Apropiadas para Cada País (NAMA) y los National Adaptation Program of Action (NAPA) ${ }^{22}$ que fomentan lógicas de coordinación y de priorización de las políticas a nivel país. En segundo lugar, para el acceso eficiente a los recursos internacionales que están disponibles se vuelve prioritario algún tipo de mecanismo nacional de coordinación, como Fondos Climáticos Nacionales o Comités de Financiamiento (Nakhooda y Jha, 2014) tal es el caso de Brasil o Indonesia, útiles para agrupar financiamiento con objetivos climáticos orientados a fortalecer la etapa de movilización de los recursos y evitar la superposición. Los mecanismos formales de financiamiento climático, en muchos casos, se encuentran en el marco de un ministerio como Unidad de Financiamiento Climático o como institución transversal a varios ministerios, tal es el caso de Argentina del Gabinete de Cambio Climático (aunque incipiente en su funcionamiento) unidad especializada que centraliza los asuntos más técnicos del cambio climático.

Todo lo anterior muestra las opciones y los límites para el manejo de la fragmentación y la falta de coordinación institucional de los recursos financieros ambientales que van desde la fusión a la extinción de fondos y a la introducción de una división más clara del trabajo en términos de coordinación temática y geográfica también útil para armonizar reglas y políticas de acceso. Es claro que existe una gran superposición de los mismos intereses muchas veces en áreas geográficas y temáticas similares, generando la duplicación de las respuestas.

Callon (1999) propuso una metáfora, la de "enmarcar" (framing), para abrir nuevos caminos de investigación. Parafraseando a Callon (1999) se puede proceder entonces a "enmarcar" formas integrales de gestión de la fragmentación de la gobernanza financiera ambiental vista como un proceso continuo de espacios de intercambio y de acción entre diversidad de actores y de valorizaciones del capital natural así como de normas y acuerdos institucionales que permitan sistematizar las barreras u obstáculos, anteriormente señaladas, proponiendo ejes de análisis que funcionen sinérgicamente y de forma transversal en todas las etapas del financiamiento climático dentro de un contexto más amplio de la investigación sobre la complejidad social y la fragmentación institucional.

Los ejes de análisis propuestos: a) políticas ambientales y proyectos, b) comercio internacional ambiental y c) mercados financieros verdes; representan una matriz de análisis que nos ayuda a revelar indirectamente procedimientos y políticas que tienen que llevarse a cabo para hacer que todas las inversiones que tienen ser hechas sean calculables en una red (Callon, 1999). Pero también buscamos incorporar un enfoque de integración

\footnotetext{
${ }^{21}$ Este enfoque, además, es un debate dado en el área internacional con el objetivo de fortalecer la gobernanza ambiental. Debate opuesto a la discusión sobre abordar el ambiente a través de una única organización. En cambio la agrupación de aumentaría la eficiencia y efectividad de los acuerdos existentes sin requerir cambios legales o administrativos de los organismos existentes.

${ }^{22}$ NAPA identifica proyectos de adaptación prioritarios para ser implementados.
} 
de las finanzas climáticas en todos sus aspectos: actores, productos, proyectos, comercio, políticas públicas; llevados a cabo por una multitud de prácticas de cálculo y gubernamentalidad que se originan tanto en el régimen de las relaciones internacionales como en la naturaleza.

Debajo se detallan la matriz de análisis exploratoria (con sus ejes propuestos) para pensar un nuevo enfoque de coordinación integral de las finanzas climáticas como conjunto de redes o dominios particulares con densidades específicas y con conexidades comunes. Pudiendo desarrollar un despliegue de la estrategia de financiamiento en cada uno de los ejes de acuerdo a las barreras específicas y al nivel de maduración que se presenta en cada uno. Como se detalla, el eje de Políticas y Proyectos está relativamente más desarrollado, pero a la vez es el que mayor confusión genera en las diferentes etapas de los fondos aunque es el más viable para lograr mayor efectividad para su implementación en cada país en el corto plazo.

Tabla 2: Matriz de análisis exploratoria

\begin{tabular}{|c|c|}
\hline $\begin{array}{c}\text { Políticas y Proyectos basados en } \\
\text { Financiamiento (Project-Based } \\
\text { Financing) }\end{array}$ & $\begin{array}{l}\text { Principales prácticas de actuación: Financiamiento } \\
\text { combinado para reducir y gestionar los riesgos; } \\
\text { Gestión de los riesgos y utilidades para incrementar } \\
\text { las oportunidades de la inversión del sector privado; } \\
\text { Implementar políticas orientadas a la transición de } \\
\text { economías bajas en carbono y resilientes al clima; } \\
\text { Desarrollar políticas de transferencia de tecnología } \\
\text { basadas en la Bioeconomía } \\
\text { Barreras: Capacidad limitada para asignar recursos } \\
\text { de manera efectiva: restricciones de capital; } \\
\text { limitaciones en el monitoreo de impactos; brechas de } \\
\text { información para el desarrollo de políticas; capacidad } \\
\text { limitada para el desarrollo de políticas de } \\
\text { financiamiento para la innovación de alto riesgo. } \\
\text { Instrumentos de coordinación: Asistencia técnica } \\
\text { para mejorar la alineación de los proyectos con los } \\
\text { criterios de elegibilidad de los fondos, las distintas } \\
\text { categorías de financiamiento y las estrategias } \\
\text { nacionales de financiamiento y políticas climáticas; } \\
\text { planificación de políticas de financiamiento como } \\
\text { incentivos de inversión ambiental privada. }\end{array}$ \\
\hline Comercio internacional climático & $\begin{array}{l}\text { Principales Prácticas: Aplicación de barreras al } \\
\text { carbono; liberación del comercio de bienes } \\
\text { ambientalmente positivos; vinculación de mercados } \\
\text { internacionalmente. } \\
\text { Barreras: tarifas reducidas de utilidades; poco } \\
\text { desarrollo de diseño de políticas específicas; } \\
\text { incertidumbre acerca de los precios y mercados del } \\
\text { carbono como reflejo de la economía real. } \\
\text { Instrumentos de coordinación: Implementar } \\
\text { comercio financiero para bienes y servicios } \\
\text { orientados a economías bajas en carbono y resilientes }\end{array}$ \\
\hline
\end{tabular}




\begin{tabular}{|l|l|}
\hline \hline Mercados Financieros verdes & $\begin{array}{l}\text { al clima; Desarrollo de políticas comerciales } \\
\text { ambientales; Vincular los mercados de exportación de } \\
\text { bienes con los mercados de carbono. }\end{array}$ \\
\hline $\begin{array}{l}\text { Principales Prácticas: Implementación de incentivos } \\
\text { para incrementar la transición de activos ecológicos; } \\
\text { Regulación incipiente de la integración de los riesgos } \\
\text { climáticos al sector financiero; Implementación de } \\
\text { impuestos al carbono; } \\
\text { Barreras: Límites institucionales y restricción de } \\
\text { capacidades; Representaciones opuestas entre las } \\
\text { percepciones del clima y el desarrollo; Experiencia y } \\
\text { capacidades limitadas y poco desarrolladas en el } \\
\text { sector específico fiscal ambiental; competencia } \\
\text { internacional en la distribución de fondos y sus } \\
\text { consecuencias como la sobrerrepresentación de } \\
\text { sectores y países } \\
\text { Instrumentos de Coordinación: asistencia técnica } \\
\text { para la implementación de gestión del conocimiento } \\
\text { en políticas de promoción de inversión financiera } \\
\text { como catalización de inversiones verdes; Asistencia } \\
\text { para la construcción de capacidades de monitoreo y } \\
\text { verificación de la información climática. }\end{array}$ \\
\hline
\end{tabular}

Fuente: Creación propia

\section{Conclusiones}

A lo largo del período que se inicia con el Plan de Acción de Bali y se refuerza con el Acuerdo de París hemos descrito una serie de desplazamientos y procesos característicos para entender la dinámica institucional que juega un papel importante en la emergencia, evolución y eventual efectividad de las instituciones de la gobernanza financiera ambiental y extraer lecciones sobre las condiciones que facilitan o impiden la coordinación entre instituciones y actores.

Abordar el problema de la adaptación y la mitigación del cambio climático requerirá que se reconsideren las trayectorias de las futuras inversiones en todos los ámbitos. El debate sobre la implementación de los flujos financieros climáticos requiere la resolución de varias problemáticas producto de la fragmentación a causa de la proliferación de fondos climáticos y la superposición de áreas y temáticas; de la falta de sinergia entre políticas de mitigación, adaptación y desarrollo y, por último, la falta de coordinación horizontal para que los mismos no se conviertan en obstáculos en sí mismos para el logro de los metas del Acuerdo de París.

Para poder analizar estas problemáticas, se desarrollaron algunas dinámicas que juegan un papel importante para la implementación de opciones de financiación para la acción climática como la creación de un marco para la gestión de los flujos de los fondos climáticos, que nos permitieron hacer, además, un balance de la situación existente y de las oportunidades y responsabilidades de desarrollo y ejecución de proyectos o políticas.

Como dinámica importante hemos querido sistematizar la configuración o mapeo de las instituciones y actores que han estado involucrados en los esfuerzos para 
invertir en soluciones al cambio climático como partes integrantes de la arquitectura financiera internacional, en el marco de la gobernanza ambiental.

Por otro lado, como hemos visto, el concepto de coordinación refiere a la necesidad de asegurar que las organizaciones e instituciones responsables del financiamiento climático global trabajen en conjunto y no dupliquen el trabajo de los demás ni permitan brechas en los servicios. A menudo, se entiende en ambos términos "horizontales", es decir, la coordinación entre organizaciones y programas, así como términos "verticales", conectar las agencias globales con las instituciones locales y los actores no estatales. Muchos estudios sobre cambio climático y finanzas concluyeron que una mejor coordinación entre agencias y partes interesadas nacionales es esencial si realmente se quiere desviar la inversión desde los negocios tradicionales (business as usual) hacia la utilización óptima de las finanzas asociadas al desarrollo compatible con el clima.

En este sentido, en un enfoque exploratorio de estrategias de coordinación horizontal y vertical, buscamos incorporar el diseño de matriz integrativa que abarque todos los aspectos de las relaciones internacionales y del ambiente para poder integrarse en el "mainstream" de políticas y decisiones de inversión de recursos ambientales y así garantizar la eficacia necesaria para cerrar la brecha existente entre finanzas y riesgos climáticos. Es así, que podemos concluir, que la eficacia para disminuir esta disparidad entre necesidades y financiamiento climático tiende a converger en la síntesis entre el resultado de estrategias de coordinación de una arquitectura fragmentada y la efectividad del monitoreo del impacto de las políticas ambientales.

Trabajar sobre diferentes aportaciones nos pueden ayudar a fomentar el debate, o buscar ampliar la gama de perspectivas y legitimidad de procesos de políticas reforzando directamente la acción coordinada para adaptarse mejor a los impactos del cambio ambiental global e investigar en qué medida las instituciones locales pueden sincronizarse con la arquitectura internacional de financiamiento climático. Es así, que a futuro se requerirán enfoques analíticos y estrategias políticas ambientales más innovadoras que nos permitan vincular en el estudio de la gobernanza ambiental global, la investigación organizacional e institucional con la preocupación central de la efectividad de las políticas ambientales y los requisitos financieros. El nuevo concepto de la "gobernanza del sistema terrestre" (Biermann, 2007) es interesante para abrirnos nuevos "marcos" de investigación. El concepto de "gobernanza del sistema terrestre" nos permitirá vincular en esta área de trabajo, los niveles globales y locales de las comunidades académicas de las ciencias naturales con el diseño y construcción de escenarios orientados por la ciencia política y relaciones internacionales.

\section{Referencias Bibliograficas}

AMERASINGHE, N., THWAITES, J, LARSEN, G. \& BALLESTEROS, A., (2017). The Future of the Funds: Exploring the Architecture of Multilateral Climate Finance. Washington DC: World Resources Institute.

BARNES, J.A. (1954). Clase y comités en una parroquia de la isla noruega. Relaciones Humanas, 7 (1), 39-58.

BECK, U. (2000). La sociedad del riesgo global. Madrid, Siglo XXI 


BIERMANN F. (2007). "Earth system governance" as a crosscutting theme of
global change research. Global Environment Change. DOI:
$\underline{10.1016 / \text { j.gloenvcha.2006.11.010 }}$

BIERMANN, F. \& PATTBERG, P. (2008). Global Environmental Governance: Taking Stock, Moving Forward. Annual Review of Environment and Resources. DOI: 10.1146/annurev.environ.33.050707.085733.

BIERMANN, F., PATTBERG, P., VAN ASSELT, H. \& ZELLI, F. (2009). The Fragmentation of Global Governance Architectures: A Framework for Analysis. Global Environmental Politics, 9 (4): 14-40.

CABRAL Y BOWLING, R. (2014). Fuentes de financiamiento para el cambio climático. Serie: Financiamiento para el Desarrollo. CEPAL, enero Recuperado de https://www.cepal.org/sites/default/files/news/files/4.r.cabral_bowling.pdf

CALLON, M. (1999). Actor network theory: The market test. En Law, J \&. Hassard, J. (Eds.), Actor network theory and after. Oxford: Blackwell, 181-195.

CEPAL (2015). La economía del cambio climático en América Latina y el Caribe: paradojas y desafíos del desarrollo sostenible (LC/G.2624), Santiago, febrero.

CMNUCC (1992). Convención Marco de las Naciones Unidas sobre el Cambio Climático (Nueva York: ONU).

CMNUCC (2016). Standing Committee on Finance. Biennial Assessment and Overview of Climate Finance Flows Report. Bonn: United Nations Framework Convention on Climate Change.

CMNUCC (2018). Biennial Assessment and Overview of Climate Finance Flows. Comité Permanente sobre Finanzas de la CMNUCC, Bonn, Alemania. Disponible en: https://unfccc.int/topics/climate-finance/resources/biennial-assessment-of-climatefinance.

CONSTANZA, R. \& DALY, H. (1992). Natural and sustainable development, Revista Conservation Biology, 6 (1), marzo (New York).

CPI CLIMATE POLICY INITIATIVE (2018). Financiamiento climático global: una visión actualizada 2018. Padraig Oliver, Alex Clark y Chavi Meattle.

CTF (2020). CTF Semi-Annual Operational Report, Ciudad de Washington.

DALY, H. (1989). Toward a Measure of Sustainable Social Net National Product, en AHMAD, Y., EL SERAFY, S. Y LUTZ, E. (eds) Environmental Accounting for Sustainable Development (Banco Mundial).

ESTENSSORO SAAVEDRA, F. (2013). La geopolítica ambiental global: el desafio del cambio climático para América latina. América Latina, Interrogantes y Perspectivas. Toronto University Press.

GLEMAREC, Y. (2011). Catalysing Climate Finance: A Guidebook on Policy and Financing Options to Support Green, Low-Emission and Climate-Resilient Development. United Nations Development Programme, New York, NY, USA.

IPCC (2018). Climate Change 2014: Synthesis Report. Contribución de los grupos de trabajo I, II, y III al Cuarto Informe de Evaluación del Grupo Intergubernamental de Expertos sobre el Cambio Climático. Ginebra, Suiza: IPCC. 
IVANOVA, M. (2007). International Environmental Governance Reform: Options and Implications. London: Royal Institute of International Affairs.

HARVEY, D. (2005). El 'nuevo' imperialismo: acumulación por desposesión, Revista Socialist Register. Buenos Aires, CLACSO.

KEOHANE, R. \& VICTOR, DAVID G. (2011), The Regime Complex for Climate Change. Perspectives on Politics, 9 (1): 7-23.

KRAMARZ, T. \& PARK, S. (2016). Accountability in Global Environmental Governance: A Meaningful Tool for Action? Global Environmental Politics. Instituto de Tecnología de Massachusetts, 16 (2). p.1-21.

KRASNER, S. (1983). International Regimes. Ithaca, NY: Cornell Univ. Press

LOCATELli, B., FEDEle, G., FAYOLlE, V. \& BAGLEE, A. (2016). Synergies between adaptation and mitigation in climate change finance. International Journal of Climate Change Strategies and Management. Vol. 8 No. 1, 112-128.

LOHMANN, L. (2009). Toward a Different Debate in Environmental Accounting: The Cases of Carbon and Cost-Benefit. Accounting, Organizations and Society. (34), 499-534.

MENDELSOHN, R., DINAR, A. \& SANGHI, A. (2001). The effect of development on the climate sensitivity of agriculture, Environment and Development Economics (6) 85-101.

MITCHELL, R.B. (2003). International environmental agreements: a survey of their features, formation, and effects. Annu. Rev. Environ. Resour. (28) 429-61.

NAJAM, A. y HALLE, M. (2010). Gobernanza ambiental global: el desafío de la rendición de cuentas. En Perspectivas del desarrollo sostenible. Boston: Universidad de Boston, Frederick S. Pardee Center for the Study of the Longer-Range Future. Disponible en: www.iisd.org/publications/pub.aspx.

NAKHOODA, S. \& JHA, V. (2014). Getting it Together: Institutional Arrangements for Coordination and Stakeholder Engagement in Climate Finance. Eschborn, Germany: GIZ.

NOGUÉ FONT, J. y VICENTE RUFÍ, J. (2001). Geopolítica, identidad y globalización. Barcelona: Ariel.

OCDE (2015). La financiación para el clima en 2013-14 y el objetivo de los 100.000 millones de dólares: Informe de la OCDE en colaboración con la Iniciativa de Política Climática, OECD Publishing, Paris. Recuperado de: https://doi.org/10.1787/9789264249974-es

OSTROM, E. (1990). Governing the Commons: The Evolution of Institutions for Collective Action, New York: Cambridge University Press.

OSTROM, E. (2010). Polycentric Systems for Coping with Collective Action and Global Environmental Change. Global Environmental Change, 20 (4): 550-557.

ROBERTS, J.T. \& WEIKMANS, R. (2017). Postface: fragmentation, failing trust and enduring tensions over what counts as climate finance. International Environmental Agreements: Politics, Law and Economics. 17, p 129-13. 
SEOANE, J. (2017). Las (re)configuraciones neoliberales de la cuestión ambiental: una arqueología de los documentos de Naciones Unidas sobre el ambiente 1972-2012, 1a ed. - Ciudad Autónoma de Buenos Aires, IEALC-Instituto de Estudios de América Latina y el Caribe, GEAL-Grupo de Estudios sobre América Latina y el Caribe.

UNEP-FI s/f “About (UNEP-FI)”. Disponible en: https://www.unepfi.org/about/.

WARREN, P., 2019. The role of climate finance beyond renewables: demandside management (DSM) and carbon capture, usage and storage (CCUS). Clim. Policy. DOI: $10.1080 / 14693062.2019 .1605330$.

YOUNG, O. (1999). Governance in World Affairs. Ithaca, NY: Cornell Univ. Press.

Cómo citar

BRUSSA, M. E. (2021). El financiamiento climático internacional y los desafíos de su catalización. Debates sobre la brecha entre necesidades climáticas y financiamiento, de Bali (2007) a París (2015). Revista de Integración y Cooperación Internacional, 32 (Ene-Jun), 22-41 